\section{(6) OPEN ACCESS}

\title{
The Denominator problem: estimating the size of local populations of men-who-have-sex-with-men and rates of HIV and other STIs in Switzerland
}

\author{
Axel Jeremias Schmidt, ${ }^{1,2}$ Ekkehardt Altpeter ${ }^{2}$
}

\begin{abstract}
- Additional material is published online only. To view please visit the journal online (http://dx.doi.org/10.1136/ sextrans-2017-053363).
\end{abstract}

${ }^{1}$ Sigma Research, Department of Public Health, Environments and Society, London School of Hygiene and Tropical Medicine, London, UK

${ }^{2}$ Communicable Diseases Division, Swiss Federal Office of Public Health, Bern, Switzerland

\section{Correspondence to} Dr Axel Jeremias Schmidt, Sigma Research, Department of Social \& Environmental Health Research, London School of Hygiene and Tropical Medicine, London WC1H 9SH, UK; axel.j. schmidt@emis-project.eu

Received 26 July 2017 Revised 11 October 2018 Accepted 15 November 2018 Published Online First 24 January 2019

\begin{abstract}
Objectives There is no estimate of the current number of men who have sex with men (MSM) in Switzerland, or of their geographical distribution. We aimed to (1) estimate MSM concentration factors and population sizes for 83 Swiss postal code areas (PCA), including the nine largest Swiss cities, and (2) calculate MSM-specific local HIV prevalence and yearly rates of diagnosed HIV, hepatitis C virus (HCV), syphilis and gonorrhoea.

Methods We triangulated data from general population estimates, MSM online surveys, published data on HIV prevalence and Swiss notification data for HIV and STDS. We compared two different formulae for the estimation of local MSM populations and calculated Bayesian 95\%-credible-intervals (Crl) for each PCA.

Results Across Switzerland, we estimate the MSM population aged 15-64 at roughly 80000 men (95\% Crl 64000-96 000). (1) MSM in Switzerland were most concentrated in the five largest cities of Zurich, Geneva, Lausanne, Bern and Basel. (2) We estimate that in 2012, 6300 MSM, or $8.0 \%$, were living with HIV, both diagnosed and undiagnosed and $1700 \mathrm{MSM}$, or 2.2\%, had non-suppressed HIV infection. Between 2010 and 2013, average yearly rates of diagnosed HIV, HCV, syphilis and gonorrhoea were $0.3 \%, 0.02 \%, 0.4 \%$ and $0.4 \%$, respectively.

Conclusions Combining general population data, MSM online surveys and notification data allows the calculation of realistic estimates of local MSM populations and thus proportions of MSM with diagnosed HIV and other STIs, with implications for prevention planning, commissioning of health services and counselling MSM on HIV/STI risk. Our methodology for Switzerland is transferable to other countries with similar data sources.
\end{abstract}

\section{INTRODUCTION}

Men who have sex with men (MSM) remain the group most affected by HIV and other STIs, but information on the size and geographical distribution of the MSM population in Switzerland and many other countries is limited. This means prevention planners and clinicians are lacking information on the burden of HIV and STIs specific to this population. While most countries share the problem of not having reliable information on local MSM denominators (cities, cantons, local authority areas, etc), Switzerland is also lacking a useful overall MSM estimate. The best available estimates for the percentage of men with same-sex sexual behaviour have been provided in secondary analyses of the quinquennial Swiss Health Survey (SHS, details-table 1): it increased from $2.8 \%$ in 2007 to $3.2 \%$ in 2012, although $95 \%$ confidence intervals for the two estimates overlap. ${ }^{12}$ However, conceptualising and querying homosexuality is complex and should take into account the broader social and cultural context, a distinction between sexual acts and sexual partners, and the recency of same-sex sexual contact. ${ }^{3}$ The SHS asked for lifetime, but not more recent, 'sexual intercourse with penetration', without mentioning non-penetrative sexual acts, oral or anal sex. The SHS measure of men-who-ever-had-penetrative-sex-with-men is thus not ideal for defining current same-sex sexual behaviour.

Alternatively, the German annual general population surveys define MSM as men with same-sex 'sexual relationships' in the last 12 months. The mean proportion $(2006-2010)^{5}$ was $2.2 \%$ for men in Germany. In France, the proportion of men reporting same-sex 'relationships/sexual intercourse' in the last 12 months was $1.4 \% .^{4}$ The restriction to 'relationships' and/or 'anal penetration' is likely to result in underestimating the prevalence of same-sex sexual contacts. In contrast, the representative British National Survey of Sexual Attitudes and Lifestyles (Natsal) defines MSM as men who, in the last 5 years, had 'sex with a man involving genital contact', based on an understanding that sex is 'oral or anal sex or any other contact involving the genital area' ${ }^{6}$ In Natsal-3, performed 2010-2012, the proportion of MSM was $2.9 \%$; also having increased over time, from $1.5 \%$ in 1990 to $2.5 \%$ in $2000 .^{7}$ It is unclear to what extent the increases in the Swiss and British surveys were due to more accurate reporting of homosexual practices and/or an increase of homosexual behaviour. ${ }^{89}$ Representative general population surveys that aim to capture the proportion with homosexual experience, desire, identity or behaviour typically have sample sizes that are too small to allow geographical breakdowns. However, there is evidence that the MSM population is not evenly distributed across cities of a given country, as many MSM migrate to larger cities that provide more gay friendly social spaces. ${ }^{10-13}$

The planning and tailoring of prevention programmes and local sexual health services requires 


\begin{tabular}{|c|c|c|c|c|c|}
\hline Country & Year & Age & Estimate $(95 \% \mathrm{Cl})$ & Definition & Time frame \\
\hline Switzerland ${ }^{1}$ & 2007 & $17-74$ & $2.8(2.2 \text { to } 3.5)^{*}$ & $\begin{array}{l}\text { Sexual intercourse with penetration (Geschlechtsverkehr mit } \\
\text { Eindringen/rapports sexuels avec pénétration) }\end{array}$ & Lifetime \\
\hline Switzerland ${ }^{2}$ & 2012 & $17-74$ & $3.2(3.0$ to 3.8$)$ & (idem) & Lifetime \\
\hline Germany $^{5}$ & 2006-2010 (yearly) & $20-59$ & 2.2 (1.6 to 2.7$)$ (mean) & Sexual relationships (Sexuelle Beziehungen) & Last 12 months \\
\hline France $^{4}$ & 2008 & $18-69$ & $1.4(1.1$ to 1.8$)$ & $\begin{array}{l}\text { Same-sex relationships/sexual intercourse } \\
\text { (Un partenaire du même sexe, des rapport sexuels) }\end{array}$ & Last 12 months \\
\hline \multirow[t]{8}{*}{$\mathrm{UK}^{7}$} & $2010-2012$ & $16-74$ & $2.6(2.1$ to 3.0$)$ & Oral or anal sex or any other contact involving the genital area & Last 5 years \\
\hline & & $16-24$ & 2.9 & & \\
\hline & & $25-34$ & 3.2 & & \\
\hline & & $35-44$ & 3.0 & & \\
\hline & & $45-54$ & 2.9 & & \\
\hline & & $55-64$ & 2.3 & & \\
\hline & & $16-64$ & 2.9 & & \\
\hline & & $65-74$ & 0.9 & & \\
\hline
\end{tabular}

*The 2007 95\% Cl for Switzerland is our own calculation based on the weighted published data.

an understanding of the population most at risk for HIV and other STIs. Therefore, in this paper, we (1) use several existing data sources to estimate the size of local MSM populations and (2) apply published data on diagnosed HIV, hepatitis C virus (HCV), syphilis and gonorrhoea to calculate yearly HIV/STI diagnosis rates, and prevalences of diagnosed and undiagnosed HIV, both locally and country-wide, specific to MSM.

\section{METHODS}

\section{Number of men in Switzerland across postal code areas}

We used data from the Swiss Federal Statistical Office to obtain the numbers of men between 15 and 64 years, broken down by postal code area (PCA). The Swiss postal code is a four-digit number, the first two digits marking the PCA. Switzerland has 83 PCAs, with the first two digits ranging from 10 to 96. Each PCA is characterised by an index city, including the nine largest Swiss cities (ie, more than 50000 inhabitants-table 2). In 2011, Switzerland had a permanent population of 7.95 million, including 2.72 million men aged 15 to $64 .{ }^{14} \mathrm{We}$ calculated the proportions of men aged 15-64 living in each PCA.

\section{Geographical concentrations of MSM in Switzerland}

We used data from the European MSM Internet Survey (EMIS2010), a pan-European 25-language survey on knowledge, needs and behaviour with regard to HIV and STIs among MSM. ${ }^{15} 16$ To be included in EMIS, respondents confirmed that they were "men who have sex with men and/or men who are attracted to other men and/or men who think they might have sex with men in the future" and were old enough to legally have sex in the country where they lived. The inclusion criteria did not state a specific recency of same-sex sexual contact.

Sexual minorities such as MSM might not be evenly distributed across a given country but concentrated in certain geographical areas. To calculate a factor for the concentration of MSM in each PCA, we divided the regional proportions of EMIS-2010 participants by the respective proportion of men in each PCA. The Swiss EMIS-2010 subsample is the largest ever sample of MSM living in Switzerland and consists of $5028 \mathrm{MSM}^{17}$ of whom $4534(90.2 \%)$ provided a postal code- $97.0 \%$ reported sex with a man in the previous 5 years. All but one (Val Bregalia, an alpine valley with 403 male residents) of the 83 Swiss PCAs were represented in EMIS-2010. To examine if the geographical distribution of the EMIS-2010 sample was representative of Swiss MSM online samples, we compared it with the geographical distribution of participants of Gaysurvey 2009 (N=1929; 1842 with PCA) and Gaysurvey 2012 (N=964; 754 with PCA). ${ }^{12} 18$

We define a local MSM concentration factor $\left(\mathrm{K}_{\mathrm{i}}\right)$ as how much more (or less) MSM concentrate in a given geographical area, compared with the national average. The following formula summarises its calculation:

$$
K_{i}=\frac{\frac{s_{i}}{s}}{\frac{p_{i}}{p}}=\frac{p \times s_{i}}{s \times p_{i}}
$$

$K$, concentration factor; $s$, number of survey participants; $p$, number of men aged $15-64 ; i$, postal code area.

\section{Two ways for estimating regional numbers of MSM}

Here, we present and compare two methods for estimating regional MSM denominators for Switzerland. We applied estimates from Natsal-3 (UK), ${ }^{7}$ as we believe Natsal's more encompassing approach to defining MSM is more likely to accurately reflect the prevalence of current same-sex behaviour in Switzerland than using the Swiss estimates of lifetime experience of same-sex penetrative intercourse.

\section{Bayesian approach using an overall MSM estimate}

To calculate the absolute numbers of MSM (15-64) per local area $\left(M_{i}\right)$, we multiplied the number of males in each PCA $\left(p_{i}\right)$ by the PCA-specific MSM concentration factor $\left(\mathrm{K}_{\mathrm{i}}\right)$ and by an estimate (g) of the national MSM proportion (2.9 from Natsal-3 for men aged 15-64-table 1). For each PCA, we calculated a Bayesian 95\%-credible-interval (CrI).

$$
\mathrm{M}_{\mathrm{i}}=\mathrm{p}_{\mathrm{i}} \times \mathrm{g} \times \mathrm{K}_{\mathrm{i}}
$$

$M$, number of MSM aged $15-64 ; p$, male permanent population aged $15-64 ; g=$ overall proportion MSM (defined as men with anal or oral sex with another man in the last 5 years); $K$, concentration factor; $i$, postal code area.

\section{Age-stratified approach}

Because the prevalence of MSM is not equally distributed across age groups, and because choice of residence is influenced by age, we performed a sensitivity analysis using an age-stratified approach to adjust for participation bias with respect to age. Again, we used estimates from Natsal-3: 2.9\% for younger age 
( $\mathrm{g}_{\mathrm{y}}, 15-24$ years), 3.5\% for medium age $\left(\mathrm{g}_{\mathrm{m}}, 25-39\right.$ years) and $2.6 \%$ for older age $\left(g_{0}, 40-64\right.$ years-table 1$)$. The respective sizes of the EMIS-2010 subsamples were 813 (748 with PCA), 2221 (2032) and 1994 (1754). We then back calculated an overall concentration factor (age-stratified approach, $\mathrm{K}_{\mathrm{i}}^{\prime}$ ).

$$
K_{i}^{\prime}=\frac{\frac{M_{i}}{M}}{\frac{p_{i}}{p}}=\frac{p \times M_{i}}{M \times p_{i}}
$$$$
M_{i}^{\prime}=p_{i y} \times g_{y} \times K_{i y}+p_{i m} \times g_{m} \times K_{i m}+p_{i o} \times g_{o} \times K_{i o}
$$

$M^{\prime}$, number of MSM aged 15-64 (age-stratified approach); $p$, male permanent population aged $15-64 ; g=$ proportion with anal or oral sex with another men in the last 5 years; $K^{\prime}$, concentration factor (age-stratified approach); $i$, postal code area; $y, 15-24$ years; $m, 25-39$ years; $o$, 40-64 years. Absolute numbers of MSM reported in this study were rounded to avoid pseudoaccuracy.

\section{Statistical methods}

We developed several hierarchical Poisson-gamma models and ran these in R-statistics. Details are described in online supplementary appendix 1 .

\section{Plausibility check}

We compared our MSM population estimates with the number of PlanetRomeo user profiles. In 2010, PlanetRomeo was the number-one online-dating platform for MSM in Switzerland. PlanetRomeo user profiles include a key city or region. To cover all languages spoken in Switzerland and available on PlanetRomeo, we searched user profiles for 'Züri(ch)/Zurigo/Zurich', 'Genève/Ginevra/Genf/Geneva', 'Bâle/Basilea/Basel', 'Lausanne/Losanna', 'Berne/Berna/Bern' and 'Lugano'. As of 14 March 2014, there were $10627 ; 3433 ; 3171 ; 2651 ; 3210$ and 741 user profiles in the respective cities and 41403 profiles in Switzerland (we ignored the possibility that some men might have more than one user profile).

\section{Data on yearly rates of diagnosed HIV, HCV, syphilis and gonorrhoea}

We extracted diagnoses of HIV infection, HCV infection, syphilis and gonorrhoea in MSM from the Swiss infectious diseases notification database (in Switzerland, laboratory information for these four infections are matched with information from clinics/ physicians, where the gender of sexual partners is reporteddata for unknown gender of sexual partners are not included in this analysis) and sorted them by PCA. To account for small numbers when broken down by 83 PCAs, we calculated the yearly mean number of notifications from 2010 to 2013, resulting in PCA-specific yearly rates of diagnoses for these infections.

\section{Data on HIV prevalence}

We based our estimates for regional HIV prevalences among MSM on two data sources: one was the publication by Kohler Schmidt et al on the HIV care cascade in Switzerland ${ }^{19}$ that used a rigorous methodology to estimate the number of HIV-diagnosed MSM retained in care in 2012. We used the same factors to back calculate the number of individuals infected with HIV, with the advantage that all back-calculation factors (retained in care $\rightarrow$ linked to care $\rightarrow$ HIV-diagnosed $\rightarrow$ HIV-infected) were MSM specific. The other data source we used was Swiss HIV notifications broken down by PCA. We assumed that the geographical distribution of HIV notifications for MSM (accumulated for 1999-2013 approximates the geographical distribution of HIV prevalence in this population). In a last step, we applied the published proportion of men with effective antiretroviral treatment $(87 \%)^{19}$ to the estimates of HIV prevalence among MSM for the largest nine index cities, to achieve the prevalence of transmissible HIV (the number of infected men minus the number of men with viral suppression).

\section{RESULTS}

MSM population concentrations varied across Switzerland, with concentration factors $\left(\mathrm{K}_{\mathrm{i}}\right)$ ranging from 0.27 to 3.85 (Bayesian approach with 95\% CrI), or from 0.17 to 3.87 (age-stratified approach, $\mathrm{K}_{\mathrm{i}}^{\prime}$ ), respectively. The highest concentrations were found in Zurich $\left(\mathrm{K}_{\mathrm{i}}=3.85\right.$; 95\% CrI, 3.58-4.13; $\left.\mathrm{K}_{\mathrm{i}}^{\prime}=3.87\right)$, followed by Basel $\left(\mathrm{K}_{\mathrm{i}}=2.37,2.08-2.69 ; \mathrm{K}_{\mathrm{i}}^{\prime}=2.46\right)$, Bern $\left(\mathrm{K}_{\mathrm{i}}=1.90,1.68-2.13 ; \mathrm{K}_{\mathrm{i}}^{\prime}=1.95\right)$, Lausanne $\left(\mathrm{K}_{\mathrm{i}}=1.86\right.$, 1.66-2.07; $\left.\mathrm{K}_{\mathrm{I}}^{\prime}=1.83\right)$ and Geneva $\left(\mathrm{K}_{\mathrm{i}}=1.18,1.06-1.32\right.$; $\left.\mathrm{K}_{\mathrm{i}}^{\prime}=1.19\right)$. No elevated concentrations were found for Lucerne $\left(\mathrm{K}_{\mathrm{i}}=0.89,0.74-1.05 ; \mathrm{K}_{\mathrm{i}}^{\prime}=0.89\right)$, Winterthur $\left(\mathrm{K}_{\mathrm{i}}=0.69,0.55\right.$ $\left.0.86 ; \mathrm{K}_{\mathrm{i}}^{\prime}=0.70\right)$, Lugano $\left(\mathrm{K}_{\mathrm{i}}=1.12,0.89-1.38 ; \mathrm{K}_{\mathrm{i}}^{\prime}=1.15\right)$ and St.Gallen $\left(\mathrm{K}_{\mathrm{i}}=1.03,0.81-1.28 ; \mathrm{K}_{\mathrm{i}}^{\prime}=1.06\right)$. Concentration factors, regional proportions of MSM and estimates of MSM population sizes for all PCAs are shown in online supplementary table s1.

Bayesian 95\% CrIs were small for all nine cities, and large only for sparsely populated rural areas figure 1 . Concentration factors based on age-stratified approaches (both frequentist and Bayesian) differed only marginally from applying an overall percentage - a proportion of 2.9\% MSM for men aged 15-64. Using other online survey data, such as Gaysurvey 2009 or 2012, resulted only in minor differences, with the exception of Lugano and other Italian-speaking areas.

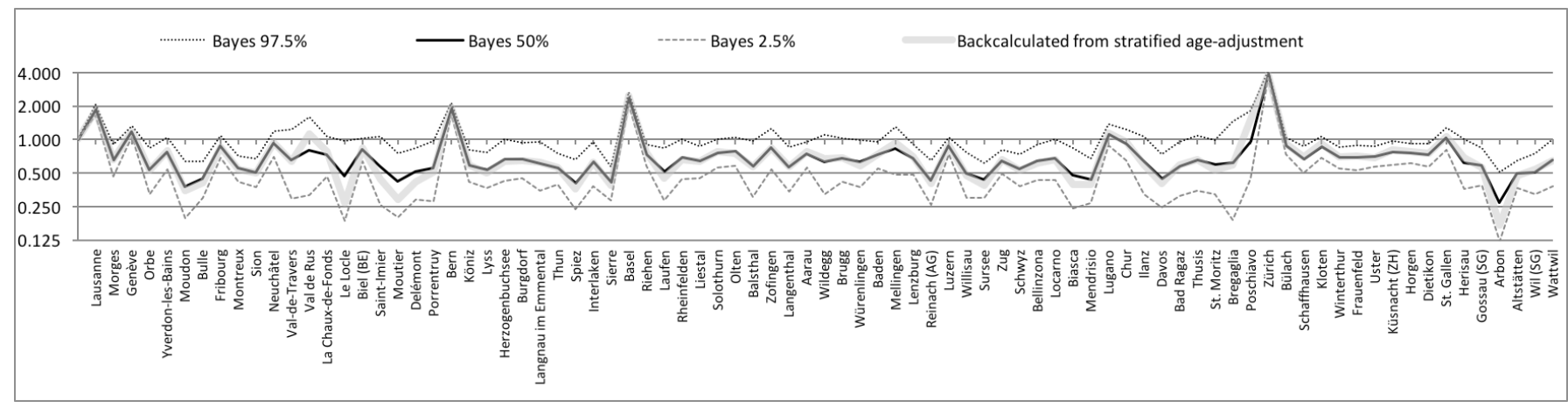

Figure 1 Comparing concentration factors (Ki), across 83 Swiss postal code areas (labelled by index cities), based on European MSM Internet Survey-2010 and Swiss Federal Statistical Office data, back calculated from age-adjusted numbers, versus Bayesian model with 95\%-credible-intervals. 


\section{Bayesian approach versus age-stratified approach}

The Bayesian model with the best fit, that is, with the lowest deviance information criterion (DIC), was the one with PCA-specific concentration factors (DIC $=573)$. Applying the same MSM concentration factor for all PCAs had the highest DIC of 2471. Using different concentration factors across both PCAs and age groups $(\mathrm{DIC}=1399)$ fitted less well: the convergence diagnostics revealed problems with the age-stratified models, indicating that the parameters were less well identified than in the model with the lowest DIC.

In absolute numbers, the largest regional MSM populations (aged 15-64), were found in the PCAs indexing Zurich (16 000; 95\% CrI: 14 300-16 500), Geneva (6000; 5400-6700), Lausanne (6000; 5200-6400), Bern (5000; 4300-5500), Basel (4000; 3400-4400), Lucerne (2300; 1900-2700) and Zürcher Unterland (PCA 81; 2100; 1800-2200). All other PCAs had MSM populations smaller than 2000. This includes three of the largest Swiss cities: Lugano, Winterthur and St.Gallen. Across Switzerland, we estimate the MSM population (aged 15-64 years; 2012) at 80000 (95\% CrI: 64 000-96 000).

\section{Plausibility check}

When comparing the number of PlanetRomeo user profiles with the estimated population sizes in six key cities and all of Switzerland, proportions of MSM with a user profile ranged from $46 \%$ in Lausanne to 58\% in Basel and 52\% overall.

\section{MSM-specific rates of HIV and STIs}

When applying Swiss notification data, estimated rates of yearly (average 2010-2013) notified HIV, syphilis and gonorrhoea varied substantially, with the highest rates in Zurich and Geneva (table 2). In Switzerland overall, HIV rates were 26 per 10000 MSM, 39 in Zurich and 33 in Geneva. HCV rates were a magnitude lower (2 per $10000 \mathrm{MSM}$ ) and showed a distinct geographical pattern, with highest rates in Lugano (6 per 10 000 MSM), suggesting transmission pathways that are different from those for HIV and bacterial STIs. Syphilis and gonorrhoea showed similar rates (Switzerland: 40 and 41, respectively, per $10000 \mathrm{MSM})$, and similar geographical patterns. Highest rates were found in Geneva (78 and 77, respectively) and Zurich (55 and 57, respectively). While syphilis testing among MSM is common in Switzerland-partly due to the availability of rapid tests-comprehensive testing for asymptomatic gonorrhoea is expensive, not covered by health insurance, and anal swabbing is rarely performed. ${ }^{20}$

When applying published data on HIV prevalence, we estimate that in $20126300 \mathrm{MSM}$, or 8.0\%, were living with diagnosed or undiagnosed HIV-12.9\% in Zurich, $12.0 \%$ in Geneva, 9.0\% in Lausanne, $8.9 \%$ in Lugano, $7.9 \%$ in Winterthur, 6.7\% in St.Gallen, 6.3\% in Basel, 6.2\% in Bern, 5.8\% in Lucerne and 5.7\% in the rest of Switzerland (figure 2). We further estimate that in 20121700 MSM, or 2.2\%, had non-suppressed HIV infection.

\section{DISCUSSION}

This is the first approach to estimating local MSM populations in Switzerland. MSM in Switzerland concentrate in the five large cities of Zurich, Geneva, Lausanne, Bern and Basel, accounting for $46 \%$ of the Swiss MSM population. In comparison, men aged 15-64 living in these five cities comprise $21 \%$ of the general male population. Our estimations allow a better understanding of the size and distribution of the Swiss MSM population and provide denominators for MSM-specific epidemiology of HIV and other STIs and the allocation of resources.

Our estimate that $11.2 \%$ of men in the city of Zurich are MSM is similar to estimates for inner London primary care trusts$11.1 \%$ in Southwark, $11.2 \%$ in Camden, $10.8 \%$ in Islington, $10.7 \%$ in Kensington and Chelsea and $15.6 \%$ in Lambeth. ${ }^{21}$

Our HIV prevalence estimate of $8.0 \%$ among MSM in Switzerland is similar to the $8.3 \%(6.1 \%-11.3 \%)$ estimate for the Netherlands. $^{22}$ Our city-specific HIV prevalences for Zurich, Geneva and Lausanne are similar to estimates for Amsterdam (13.7\%) and Rotterdam (11.0\%), ${ }^{22}$ inner London $(9.5 \%),{ }^{21}$ Berlin $(8.2 \%$ 14.2\%), Hamburg (8.2\%-16.5\%), Munich (7.4\%-10.9\%), Frankfurt (8.0\%-13.1\%) and Cologne (5.7\%-8.2\%), ${ }^{23}$ though using slightly different approaches and slightly different age groups.

STI diagnosis rates among MSM were between one and two magnitudes higher than in the general population, when comparing with 2012 notification data ${ }^{24}$ among non-MSM ${ }^{25}$ : HIV, 26/10 000 vs $0.4 / 10000$; syphilis, $40 / 10000$ vs $0.3 / 10$ 000; gonorrhoea, 41/10 000 vs 1.5/10 000 .

MSM in Zurich (and Switzerland ${ }^{16}$ had a 10 -fold lower likelihood of receiving an anal swab as part of routine STI testing than their counterparts in London, Amsterdam, Dublin, Oslo or Stockholm-even when controlling for the number of sexual partners. ${ }^{20}$ Asymptomatic rectal gonorrhoea and chlamydia are common in this population. ${ }^{26-28}$ If testing standards in

\begin{tabular}{|c|c|c|c|c|c|c|c|}
\hline PCA & Index city & HIV/year & HCV/year & Syphilis/year & Gonorrhoea/year & Prevalent HIV & Infectious HIV \\
\hline 12 & Geneva & $33(29-36)$ & $2.9(2.6-3.3)$ & $78(70-87)$ & 77 (69-86) & $12.0(10.8-13.4)$ & $3.3(2.9-3.7)$ \\
\hline 40 & Basel & $16(14-19)$ & $0.6(0.6-0.7)$ & $31(27-35)$ & $29(26-33)$ & $6.3(5.6-7.3)$ & $1.7(1.5-2.0)$ \\
\hline 10 & Lausanne & $23(21-26)$ & $2.6(2.3-2.9)$ & $50(45-56)$ & $52(46-58)$ & $9.0(8.1-10.1)$ & $2.5(2.2-2.7)$ \\
\hline 60 & Lucerne & $22(19-26)$ & $3.3(2.8-4.0)$ & $30(25-36)$ & $24(21-29)$ & $5.8(4.9-7.0)$ & $1.6(1.3-1.9)$ \\
\hline 90 & St.Gallen & $16(13-20)$ & $2.0(1.6-2.6)$ & $20(16-26)$ & $10(08-13)$ & $6.7(5.3-8.4)$ & $1.8(1.5-2.3)$ \\
\hline \multirow[t]{3}{*}{69} & Lugano & $17(14-21)$ & $5.7(4.6-7.2)$ & $27(22-33)$ & $21(17-26)$ & $8.9(7.3-11.2)$ & $2.4(2.0-3.1)$ \\
\hline & All other PCAs & 23 & 2.0 & 24 & 33 & 5.7 & 1.6 \\
\hline & Switzerland & 26 & 2.8 & 40 & 41 & 8.0 & 2.2 \\
\hline
\end{tabular}

gonorrhoea/year, notified diagnoses of gonorrhoea, mean 2010-2013; HCV/year, notified diagnoses, mean 2010-2013; HIV/year, notified diagnoses, mean 2010-2013; MSM, men who have sex with men; PCA, postal code area; infectious HIV, prevalence of non-suppressed HIV (undiagnosed, untreated, not effectively treated); prevalent HIV, prevalence of HIV (diagnosed and undiagnosed); syphilis/year, notified diagnoses, mean 2010-2013. 


\section{Estimated MSM population aged 15-64 (bubble size), and HIV prevalence}

$0<3.5 \%$

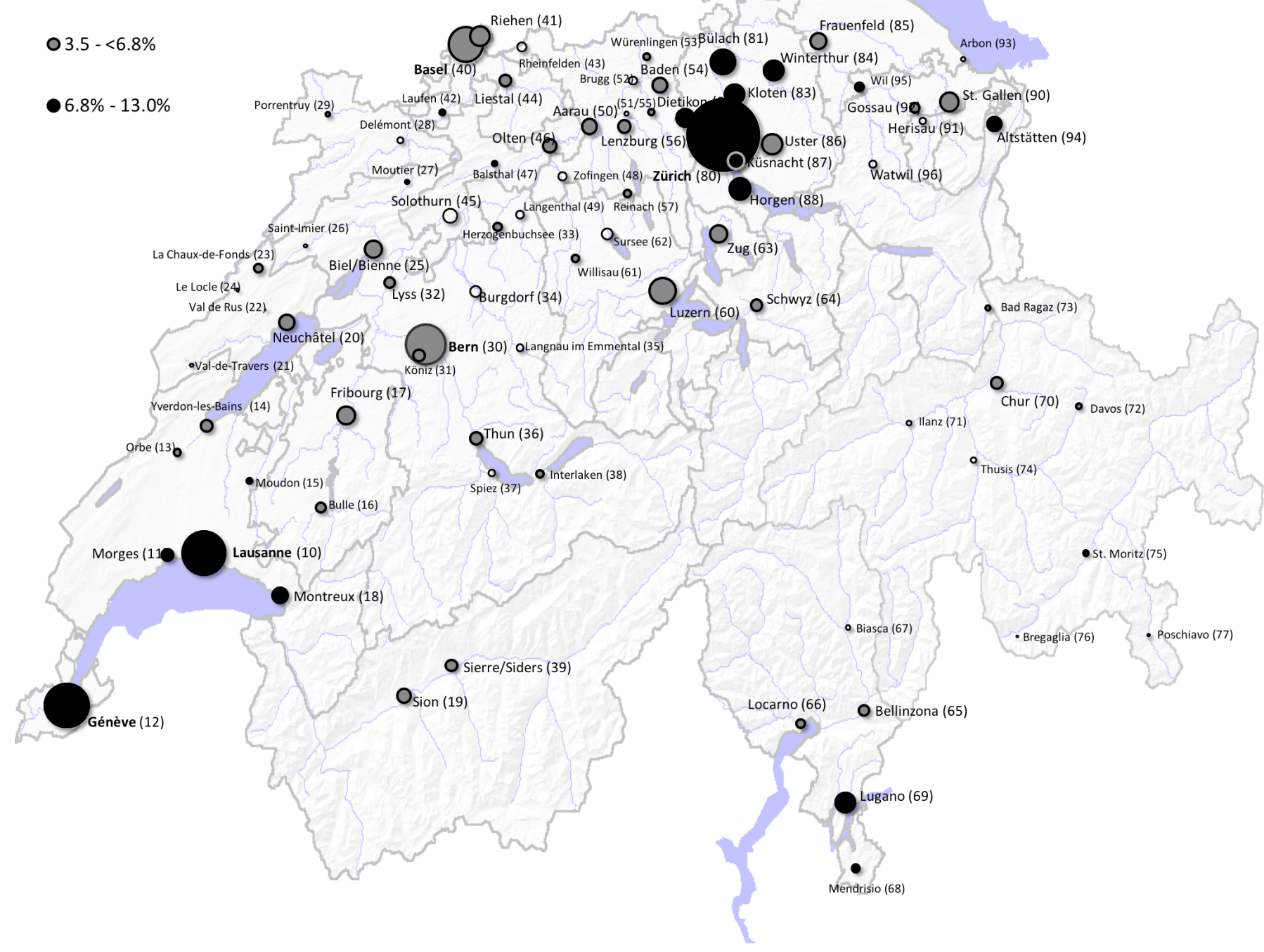

Figure 2 Estimated MSM population aged 15-64 (bubble size) and HIV prevalence (shading). MSM, men who have sex with men.

Switzerland were as comprehensive as in the UK, rates of diagnosed gonorrhoea would be twice as high as reported here.

\section{Limitations}

There are several limitations to our study. First, we used British and not Swiss overall and age-specific MSM estimates, as no comparable Swiss data were available. Even across Western European countries, the proportion of men engaging in same-sex sexual contacts may differ. However, applying other European MSM population estimates (instead of Natsal-3) has no influence on the geographical distribution and the concentration factors across Switzerland, and the magnitude of the estimated HIV prevalence and STI incidence would not change. Second, the Bayesian model may be challenged: in the JAGS model, for the overall proportion of MSM in Switzerland, we assumed a binomial distribution with a non-informative beta prior. This estimate was the basis for the estimation of the concentration factor in each PCA. We neglected spatial correlation which may be justified by the fact that the neighbourhood of a PCA has no role in the estimation of the proportion of MSM. Furthermore, convergence diagnostics and the DIC revealed that the model with a differential concentration factor across PCAs fitted best and converged better than the more complex models. Third, the geographical distribution of MSM used in this study is strictly speaking the distribution of MSM internet survey participants. Given the broad coverage of the Swiss population with Internet access in 2010, and given that EMIS-2010 was offered in 25 languages, including all three major official languages in Switzerland, we think that any impact on true geographical distribution is negligible. The systematic differences we found for Italian-speaking regions of Switzerland are likely to be explained by EMIS encouraging Italian speakers by providing an Italian language version, while the Gaysurvey has always been available only in French and German. Fourth, using PCAs rather than municipalities introduces a systematic bias in capturing some cities, but it does not affect the estimates for yearly HIV/STI diagnoses rates or prevalence, as the bias is the same in both numerator and denominator. When calculating concentrations factors, it is important to point out that for example, "Zurich" is actually the PCA number 80 , that is the city of Zurich and 
not all the city's urban area. Fifth, our assumption that the geographical distribution of MSM diagnosed with HIV in Switzerland 1999-2013 approximates the geographical distribution of HIV prevalence may be challenged, however, we believe that mobility after HIV diagnosis is low and access to HIV-testing and HIV-treatment services are similar for MSM in Switzerland. Sixth, using telephone interviews in the general population to capture homosexuality has been critised. ${ }^{3}$ Other methods, based on self-reported HIV diagnoses in online surveys and notification data plus household internet access, have resulted in a smaller population size of about $70000 \mathrm{MSM}^{29}$; however, this is still within our credible interval. Seventh, the age limitation 15-65 for being sexually active can be questionned. As for the lower bound; MSM living in Switzerland (2010) reported a median age at first sex with a man of 18 years, with an IQR of 15-21. For this reason, we think that MSM younger than 15 years can be neglected for this analysis. As for the upper bound; when further applying the Natsal-3 estimate of $0.9 \%{ }^{7}$ for men aged $65+$ (table 1), the number of all MSM in Switzerland can be estimated at 85000 (95\% CI 70000 to 100 000. Despite these limitations, we believe our findings to be sufficiently reliable for the commissioning of health services.

\section{Implications}

Our study has implications for the commissioning of STI services as well as for the recommendation for HIV oral chemoprophylaxis, also known as pre-exposure prophylaxis (PrEP). We found that between 2010 and 2013, 0.26\% in Switzerland, 0.33\% in Geneva and $0.39 \%$ of MSM in Zurich had been diagnosed with HIV every year (in a population with increasing rates of HIV testing), ${ }^{24}$ and yearly HIV diagnoses among MSM in Switzerland have since decreased. ${ }^{30}$ The WHO recommends PrEP for MSM with substantial risk of infection, defined as an HIV incidence of about 3\% or higher. ${ }^{31}$ This means that in Switzerland, PrEP should only be recommended to a subset of MSM. Our data also suggest that in areas with concentrated MSM populations, risk for STI acquisition is very high. Low-threshold targeted comprehensive STI testing is warranted. Our study also has implications for counselling on HIV risk. While in the general population about one in a thousand men and women in Switzerland has HIV, ${ }^{19}$ it is about 1 in 10 among MSM. Gay and other MSM need to be aware that on average, one in 45 sexual partners can transmit HIV (table 2).

\section{CONCLUSIONS}

Combining general population data, MSM online surveys and notification data provides realistic estimates of local MSM

Key messages

- Precise population sizes of sexual minorities such as male homosexuals or men-who-have-sex-with-men (MSM) are difficult to calculate, due to variations of sociocultural contexts and definitions.

- Triangulating general population, MSM online survey and notification data allows the calculation of rough but realistic estimates of local MSM populations and thus MSM-specific rates of HIV and other STIs.

- MSM-specific prevalence of HIV and incidence of diagnosed HIV, hepatitis C virus, syphilis and gonorrhoea vary substantially across regions and cities within Switzerland.

- Our methodology can be adapted to other countries with similar data sources. population sizes and thus MSM-specific rates of diagnosed HIV and other STIs. Our methodology can be adapted to other countries with similar data sources; for example, for most European countries, data on the geographical distribution of MSM online survey participants is available. ${ }^{1632}$

\section{Handling editor Prof Nicola Low}

Acknowledgements The authors want to thank all gay and bisexual men in Switzerland who participated in the European MSM Internet Survey (EMIS) and the Swiss Gaysurvey; PlanetRomeo for invaluable support with recruiting gay and other MSM for online surveys; Stéphanie Lociciro and André Jeannin (IUMSP, Lausanne, Switzerland) for providing PCA data from Gaysurvey 2009 and 2012; Ursula von Rüden (Federal Centre of Health Education, Cologne, Germany) for the regular provision of estimates for the German MSM population), Martin Gebhardt und JeanLuc Richard (FOPH, Bern, Switzerland) for providing numerator data (1999-2013 for HIV, HCV, syphilis and gonorrhoea); Andreas Birrer (FOPH, Bern, Switzerland) for providing geo-coordinates of Swiss cities and municipalities; Caoimhe Cawley (Robert Koch Institute, Berlin, Germany) for proof-reading the manuscript, Peter Weatherburn and David Reid (Sigma Research, London) for invaluable input at the revision level and Michael Bochow (sociologist, Berlin, Germany) for a decade of guidance on MSM populations.

Contributors AJS: designed the study, performed all calculations except Bayesian analyses, co-ordinated the EMIS project and wrote the manuscript. EA: performed the Bayesian analyses and contributed to the manuscript.

Funding EMIS-2010 was funded by the Executive Agency for Health and Consumers, EU Health Programme 2008-2013 (funding period: 14 March 2009 to 13 September 2011).

Competing interests None declared.

Patient consent for publication Not required.

Provenance and peer review Not commissioned; externally peer reviewed.

Open access This is an open access article distributed in accordance with the Creative Commons Attribution Non Commercial (CC BY-NC 4.0) license, which permits others to distribute, remix, adapt, build upon this work non-commercially, and license their derivative works on different terms, provided the original work is properly cited, appropriate credit is given, any changes made indicated, and the use is non-commercial. See: http://creativecommons.org/licenses/by-nc/4.0/

\section{REFERENCES}

1 Jeannin A, Meystre-Agustoni G, Lociciro S. Système de suivi de la stratégie de lutte contre le VIH/sida en Suisse : rapport de synthèse 2004-2008 [Monitoring system for the HIVIAIDS strategy in Switzerland. synthesis report 2004-2008]. Lausanne: Institut universitaire de médecine sociale et préventive (IUMSP). 2010. Available: https:// serval.unil.ch/resource/serval:BIB_F8240BA3DE56.P001/REF

2 Lociciro S, Simonson T, Samitca S, et al. Système de surveillance du VIH et des IST de deuxième génération en Suisse. Rapport de synthèse 2012-2016 [Second-generation HIV and STI surveillance system in Switzerland. Synthesis report 2012-2016]. Lausanne: institut universitaire de médecine sociale et préventive (IUMSP). 2016. Available: https://www.iumsp.ch/Publications/pdf/rds278_fr.pdf

3 Michaels S, Lhomond B. Conceptualization and measurement of homosexuality in sex surveys: a critical review. Cad Saude Publica 2006;22:1365-74.

4 Bajos N, Bozon M. Enquête sur la sexualité en France. Pratiques, Genre et Santé. Paris: [Sexuality in France. Practices, Gender and Health]Éditions La Découverte, 2008.

5 Von Rüden U. Public Awareness of AIDS in the Federal Republic of Germany. Knowledge, attitudes and behaviour relating to protection against AIDS. A repeat survey by the Federal Centre for Health Education (BZgA). Cologne, 2007, 2008, 2009, 2010, 2011.

6 Mercer CH, Fenton KA, Copas AJ, et al. Increasing prevalence of male homosexual partnerships and practices in Britain 1990-2000: evidence from national probability surveys. AIDS 2004;18:1453-8.

7 Mercer $\mathrm{CH}$, Tanton C, Prah P, et al. Changes in sexual attitudes and lifestyles in Britain through the life course and over time: findings from the National Surveys of Sexual Attitudes and Lifestyles (Natsal). Lancet 2013;382:1781-94.

8 Twenge JM, Sherman RA, Wells BE. Changes in American adults' reported same-sex sexual experiences and attitudes, 1973-2014. Arch Sex Behav 2016;45:1713-30.

9 Bajos N, Bozon M, Beltzer N, et al. Changes in sexual behaviours: from secular trends to public health policies. AIDS 2010;24:1185-91.

10 Wimark T, Östh J. The city as a single gay male magnet? Gay and lesbian geographical concentration in Sweden. Popul Space Place 2014;20:739-52.

11 Marcus U, Schmidt AJ, Hamouda O. Estimating the regional distribution of men who have sex with men (MSM) based on Internet surveys. BMC Public Health 2009;9:181.

12 Lociciro S, Jeannin A, Dubois-Arber F. Les comportements face au VIH/Sida des hommes qui ont des relations sexuelles avec des hommes. Enquête Gaysurvey 2012 
[Behaviours of men who have sex with men in times of HIV/AIDS]. Lausanne: Institut universitaire de médecine sociale et préventive (IUMSP), 2013.

13 Black D, Gates G, Sanders S, et al. Why do gay men live in San Francisco? J Urban Econ 2002;51:54-76.

14 Swiss Federal Statistical Office. Switzerland's population 2011. Neuchâtel: Swiss Federal Statistical Office, 2012

15 Weatherburn P, Schmidt AJ, Hickson F, et al. The European Men-who-have-sex-withmen Internet Survey (EMIS): design and methods. Sexuality Research and Social Policy 2013;10:243-57.

16 The EMIS Network. EMIS 2010: The European men-who-have-sex-with-men internet survey. Findings from 38 countries. Stockholm: European Centre for Disease Prevention and Control (ECDC), 2013.

17 Lociciro S, Jeannin A, Dubois-Arber F. Les hommes qui ont des relations sexuelles avec des hommes. Résultats de l'enquête EMIS 2010. Lausanne: Institut universitaire de médecine sociale et préventive (IUMSP), 2012.

18 Lociciro S, Jeannin A, Dubois-Arber F. Les comportements face au VIH/SIDA des hommes qui ont des relations sexuelles avec des hommes. Résultats de Gaysurvey 2009 [Behaviours of men who have sex with men in times of HIVIAIDS]. Lausanne: Institut universitaire de médecine sociale et préventive (IUMSP), 2010.

19 Kohler P, Schmidt AJ, Cavassini M, et al. The HIV care cascade in Switzerland: reaching the UNAIDS/WHO targets for patients diagnosed with HIV. AIDS 2015;29:2509-15.

20 Schmidt AJ, Hickson F, Weatherburn P, et al. Comparison of the performance of STI screening services for gay and bisexual men across 40 European cities: results from the European MSM Internet Survey. Sex Transm Infect 2013;89:575-82.

21 Ruf M, Delpech V, Osuagwu U, et al. Men who have sex with men: estimating the size of at-risk populations in London primary care trusts. Int I STD AIDS 2011;22:25-9.

22 Op de Coul ELM, Schreuder I, Conti S, et al. Changing patterns of undiagnosed HIV Infection in the Netherlands: who benefits most from intensified hiv test and treat policies? PLoS One 2015;10:e0133232.

23 Marcus U, Schmidt AJ, Kollan C, et al. The denominator problem: estimating MSM-specific incidence of sexually transmitted infections and prevalence of HIV using population sizes of MSM derived from Internet surveys. BMC Public Health 2009;9:181.

24 Swiss Federal Office of Public Health. VIH, syphilis, gonorrhée et chlamydiose en Suisse en 2015 : survol épidémiologique [HIV, syphilis, gonorrhoea, and chlamydiosis in Switzerland, 2015. An epidemiological overview]. Bulletin 2016;46:12-36.

25 Swiss Federal Office of Public Health. Nombre de cas de VIH et d'IST en 2012: rapport, analysees et tendances [HIV and STIs in Switzerland - Analyses and Trends 2012]. . Bulletin, 2013: 22. 363-87.

26 Dudareva-Vizule S, Haar K, Sailer A, et al. Prevalence of pharyngeal and rectal Chlamydia trachomatis and Neisseria gonorrhoeae infections among men who have sex with men in Germany. Sex Transm Infect 2014;90:46-51.

27 Kent CK, Chaw JK, Wong W, et al. Prevalence of rectal, urethral, and pharyngeal chlamydia and gonorrhea detected in 2 clinical settings among men who have sex with men: San Francisco, California, 2003. Clin Infect Dis 2005;41:67-74.

28 Annan NT, Sullivan AK, Nori A, et al. Rectal chlamydia-a reservoir of undiagnosed infection in men who have sex with men. Sex Transm Infect 2009;85:176-9.

29 Marcus U, Hickson F, Weatherburn P, et al. Estimating the size of the MSM populations for 38 European countries by calculating the survey-surveillance discrepancies (SSD) between self-reported new HIV diagnoses from the European MSM internet survey (EMIS) and surveillance-reported HIV diagnoses among MSM in 2009. BMC Public Health 2013;13:919.

30 Chapin-Bardales J, Schmidt AJ, Guy RJ, et al. Trends in human immunodeficiency virus diagnoses among men who have sex with men in North America, Western Europe, and Australia, 2000-2014. Ann Epidemiol 2018;28:874-80.

31 World Health Organisation (WHO). Policy brief on oral pre-exposure prophylaxis of HIV infection (PrEP). 2015. Available: http://www.who.int/hiv/pub/prep/policy-briefprep-2015/en

32 EMIS-2017. The (2nd) European MSM Internet Survey. London, 2017. www. emis2017.eu. 